\title{
Basal-flow characteristics of a linear medium sliding frictionless over small bedrock undulations
}

\author{
G. Hilmar Gudmundsson \\ Versuchsanstalt für Wasserbau, Hydrologie und Glaziologie, ETH Zentrum, Gloriastrasse 37/39, CH-8092 Zürich, Switzerland
}

\begin{abstract}
The basal deformation of a gravity-driven linear creeping flow sliding frictionless over slowly varying bed undulations in two dimensions is analysed analytically, using results from second-order perturbation theory. One of the key results is that, close to sinusoidal bedrock undulations, up to two different spatial regions of local extrusion flow may arise. The offset and onset of extrusion flow is controlled primarily by the amplitude-to-wavelength ratio. Above the crest of a sinusoidal bed line, a local maximum of the surface-parallel velocity develops for $\varepsilon:=a k<0.138$, where $a$ is the amplitude and $k$ is the wave number. As $\varepsilon$ increases from zero to this critical value, the vertical position of the velocity maximum moves from $k z=1$ to $k z \approx 1.98$, where $z$ is the vertical distance above the mean bed line. Within and above the trough of a sinusoid, a region of local minimum of the surface-parallel velocity component develops, which shifts from $k z=1$ towards the bed line as $\varepsilon$ increases from zero to $\frac{1}{2}$. Below this velocity minimum, and for some distance above the velocity maximum, the surface-parallel velocity increases with depth. This type of extrusion flow will cause a reversal of borehole-inclination profiles close to the bedrock.
\end{abstract}

\section{INTRODUGTION}

Most of the internal deformation of glaciers takes place in a relatively narrow region close to the base. One must, in general, expect small local bedrock undulations, which protrude into the ice, to affect the basal flow and possibly to cause a flow pattern considerably different from the one predicted by the well-known plane-slab solution. Knowledge of the flow perturbations associated with bedrock undulations is, among other things, important for the interpretation of slope measurements in ice, and because of their possible effect on ice stratigraphy.

This paper develops an analytical solution for a highly viscous medium flowing over a perfectly lubricated sinusoidal bed and analyses its properties. A numerical treatment of the problem for high roughness values, where the roughness $r$ is defined as the ratio of the bed's amplitude $a$ to its wavelength $\lambda(r:=a / \lambda)$ and using Glen's flow law, is the subject of a further paper (Gudmundsson, 1997).

\section{NOTATION}

$A_{\mathrm{m}} \quad$ Morland's $A$ parameter, defined through Equation (18)

a
Amplitude of a sinusoidal wave

Clausius-Clapeyron constant

Acceleration of gravity

Mean glacier thickness

Wave number

Controlling wave number, defined by

Equation (17)

Thermal conductivity

Latent heat of fusion per unit volume of ice

Set of integers

\section{$p \quad$ Mean normal pressure \\ $p_{\text {a }} \quad$ Atmospheric pressure \\ $p_{\infty} \quad$ Overburden pressure \\ $r \quad$ Roughness; $r:=a / \lambda$}

$s(\varepsilon, \delta, n)$ Sliding function;

$$
s(\varepsilon, \delta, n):=\varepsilon^{n+1} k u_{\mathrm{b}}(\varepsilon, \delta, n) /\left(2 A \tau_{\mathrm{b}}{ }^{n}\right)
$$

$u_{\mathrm{b}} \quad$ Basal sliding velocity

$U_{\mathrm{b}} \quad$ Non-dimensional sliding velocity,

$$
U_{\mathrm{b}}=A \tau_{\mathrm{b}}{ }^{n} \lambda U_{\mathrm{b}}
$$

$u_{\mathrm{s}} \quad$ Surface velocity

$U_{\pi / 2}^{\max } \quad$ The local maximum of $v_{x}$ at $k x=\pi / 2$

$U_{\pi / 2}^{\text {saddle }} \quad$ The saddle point of $v_{x}$ at $k x=\pi / 2$

$U_{3 \pi / 2}^{\min } \quad$ The local minimum of $v_{x}$ at $k x=3 \pi / 2$

$V_{X}, V_{Z} \quad$ Scaled velocities, defined as

$\left(V_{X}, V_{Z}\right):=\left(v_{x}, v_{z}\right) / u_{\mathrm{b}}$

$v_{i} \quad$ Components of the velocity vector

$\bar{w} \quad \bar{w}:=\lambda / \lambda_{*}=k_{*} / k$

$x, y, z \quad$ Space coordinates

$X, Z \quad$ Scaled coordinates; $(X, Z)=k(x, z)$

$Z_{\text {crit }} \quad$ The $Z$ coordinate of $U_{\pi / 2}^{\text {saddle }}$ and $U_{\pi / 2}^{\max }$ at $\varepsilon=\varepsilon_{\pi / 2}^{\text {crit }}$

$z_{0} \quad$ Vertical position of glacier bed

$\alpha \quad$ Mean surface slope

$\beta(x) \quad$ Local bedrock slope; $\tan \beta(x)=\mathrm{d} z_{0}(x) / \mathrm{d} x$

$\beta_{1} \quad \beta_{1}:=\bar{w}^{2} /\left(\bar{w}^{2}+1\right)$

$\delta \quad$ Thinness parameter; $\delta:=(k h)^{-1}$

$\delta_{i j} \quad$ Kronecker delta

$\dot{\epsilon}_{\mathrm{II}} \quad$ Second invariant of the strain-rate tensor; $\dot{\epsilon}_{\mathrm{II}}:=\frac{1}{2} \dot{\epsilon}_{i j} \dot{\epsilon}_{i j}$

$\dot{\epsilon}_{i j} \quad$ Components of strain-rate tensor

$\dot{\epsilon} \quad$ Effective strain rate; $\dot{\epsilon}:=\sqrt{\frac{1}{2} \dot{\epsilon}_{i j} \dot{\epsilon}_{i j}}$

$\varepsilon \quad$ Local bed-slope parameter, $\varepsilon:=a k$

$\varepsilon_{\pi / 2}^{\text {crit }} \quad$ For $\varepsilon<\varepsilon_{\pi / 2}^{\text {crit }}, v_{x}(x, z)$ has a stationary point

somewhere along the vertical line $k x=\pi / 2$ 
$\varepsilon_{3 \pi / 2}^{\text {crit }} \quad$ For $\varepsilon<\varepsilon_{3 \pi / 2}^{\text {crit }}, v_{x}(x, z)$ has a stationary point somewhere along the vertical line $k x=3 \pi / 2$

$\eta \quad$ Viscosity

$\lambda \quad$ Wavelength

$\lambda_{*} \quad$ Transition wavelength

$\rho \quad$ Specific density

$\sigma_{\text {II }}^{\prime} \quad$ Second deviatoric stress invariant;

$$
\sigma_{\mathrm{II}}^{\prime}:=\frac{1}{2} \sigma_{i j}^{\prime} \sigma_{i j}^{\prime}
$$

$\sigma_{i j} \quad$ Components of the stress tensor

$\sigma_{i j} \quad$ Components of the deviatoric stress tensor;

$$
\sigma_{i j}^{\prime}:=\sigma_{i j}-\frac{1}{3} \delta_{i j} \sigma_{k k}
$$

$\tau$

$\tau_{\mathrm{b}}$

Effective stress; $\tau:=\sqrt{\sigma_{\mathrm{II}}^{\prime}}$

Suffixes

I Properties of ice

B Properties of bedrock

b Basal properties

Dimensional quantities are usually in lower-case letters and non-dimensional quantities are in capital letters.

\section{PREVIOUS WORK}

Theoretical treatment of flow over undulating bed is difficult and only a few analytical solutions exist (Nye, 1969, 1970; Kamb, 1970; Morland, 1976a, b; Fowler, 1979, 1981). These solutions often apply to somewhat idealized conditions at the glacier bed but nevertheless give a valuable insight into the nature of the flow. Numerical work has so far been limited to a few cases (Meyssonnier, 1983; Schweizer, 1989; Schweizer and Iken, 1992; Raymond, unpublished).

Nye (1969) and Kamb (1970) found an approximate solution for a highly viscous Newtonian fluid sliding over a perfectly lubricated bed. They used a perturbation approach and calculated the flow field to first order in $\varepsilon:=a k$, where the vertical position of the bed line is given by $z_{0}=$ $a \sin k x$.

Ignoring the effect of regelation and assuming no tangential traction, the boundary conditions along the bed line are

$$
-v_{x} \frac{\mathrm{d} z_{0}}{\mathrm{~d} x}+v_{z}=0, \quad \text { on } \quad z=z_{0},
$$

and

$$
\sigma_{x z}=\frac{1}{2}\left(\sigma_{x x}-\sigma_{z z}\right) \tan 2 \beta(x), \quad \text { on } \quad z=z_{0},
$$

where $\tan \beta(x):=\mathrm{d} z_{0}(x) / \mathrm{d} x$. The problem is depicted in Figure 1 and the variables have been defined above under Notation.

The field perturbations are to first order (Nye, 1969; Kamb, 1970):

$$
\begin{gathered}
v_{x}(x, z)=u_{\mathrm{b}}+u_{\mathrm{b}} a k^{2} z \mathrm{e}^{-k z} \sin k x+O\left(\varepsilon^{2}\right), \\
v_{z}(x, z)=u_{\mathrm{b}} k(1+k z) \mathrm{e}^{-k z} a \cos k x+O\left(\varepsilon^{2}\right), \\
p(x, z)=p_{\infty}+2 \eta u_{\mathrm{b}} k^{2} \mathrm{e}^{-k z} a \cos k x+O\left(\varepsilon^{2}\right), \\
\sigma_{x x}^{\prime}(x, z)=-\sigma_{z z}^{\prime}=2 \eta u_{\mathrm{b}} k^{3} z \mathrm{e}^{-k z} a \cos k x+O\left(\varepsilon^{2}\right), \\
\sigma_{x z}(x, z)=-2 \eta u_{\mathrm{b}} k^{3} z \mathrm{e}^{-k z} a \sin k x+O\left(\varepsilon^{2}\right), \\
\tau(x, z)=2 \eta u_{\mathrm{b}} a k^{3} z \mathrm{e}^{-k z}=2 \tau_{\mathrm{b}} \frac{z}{a} \mathrm{e}^{k z}+O\left(\varepsilon^{2}\right),
\end{gathered}
$$

where $\tau:=\sqrt{\sigma_{\mathrm{II}}^{\prime}}$, which is sometimes called the effective stress, $p_{\infty}$ is the pressure applied at the upper boundary of the medium, $\sigma_{i j}$ are the components of the stress tensor and $\sigma_{i j}^{\prime}$ are the components of the deviatoric stress tensor. The driving force of the motion is a constant shear stress applied at the upper boundary. The basal sliding velocity, $u_{\mathrm{b}}$, is given by

$$
u_{\mathrm{b}}=\frac{\tau_{\mathrm{b}}}{\eta a^{2} k^{3}},
$$

where $\eta$ is the viscosity of the ice, and $\tau_{\mathrm{b}}$ the driving stress.

Expressions (3a)-(3f) display some interesting features. One of them is the fact that $\tau$, in Equation (3f), shows no dependence on $x$. This will of course also apply to the second invariant of the strain-rate tensor. Another interesting feature of the linear solutions given above is the occurrence of extrusion flow, which is here defined as an increase of the horizontal flow-velocity component with depth. At the point $k x=3 \pi / 2+2 \pi l$ where $l \in N$, where $N$ is the set of integers and $z=z_{\min }:=1 / k, v_{x}$ has a local minimum:

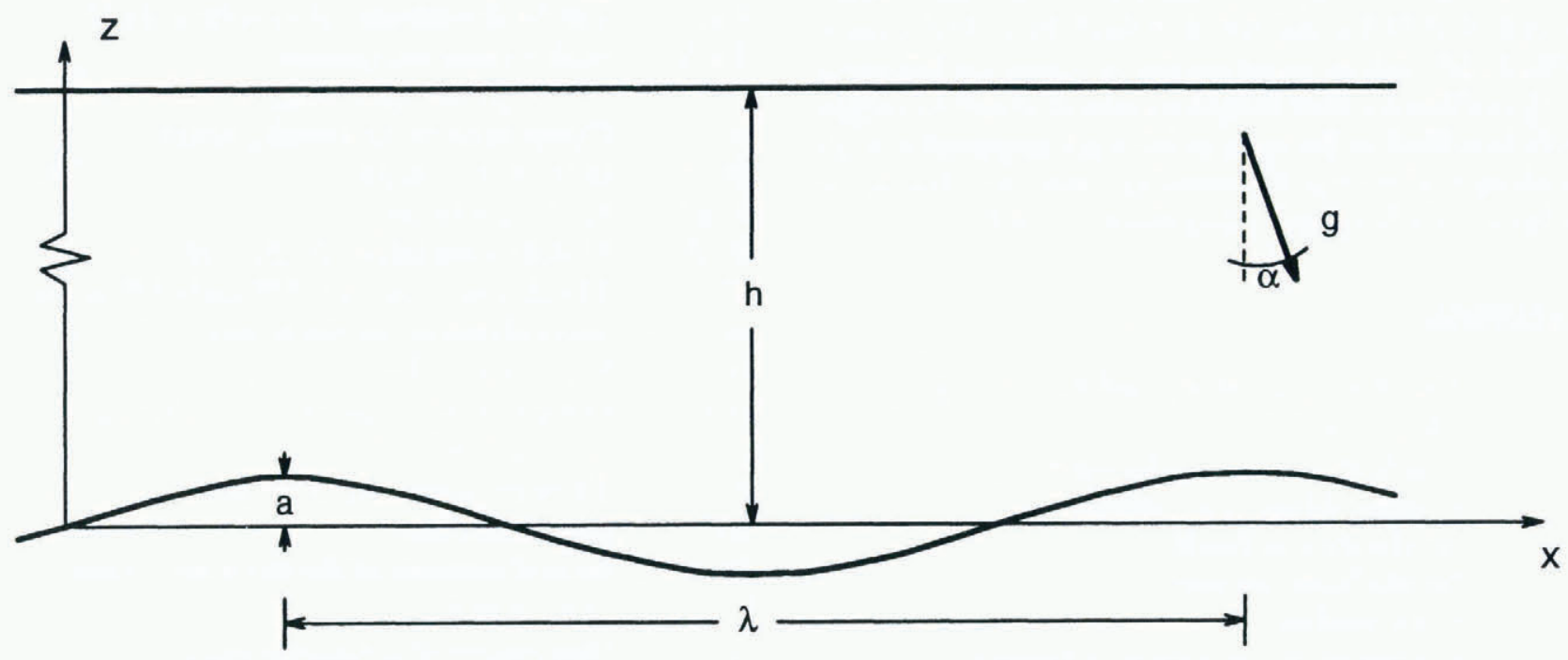

Fig. 1. Flow over a sinusoidal bed. The coordinate system makes the angle $\alpha$ with respect to the horizontal. The vertical position of the bed line $z_{0}$, is $z_{0}=a \sin k x$. The sine wave has the wavelength $\lambda=2 \pi / k$ and amplitude $a$. The surface velocity is denoted by $u_{\mathrm{s}}$ and the sliding velocity by $u_{\mathrm{b}}$. $h$ is the glacier thickness. 


$$
v_{\min }:=v_{x}\left(x=\frac{3 \pi}{2 k}, z=\frac{1}{k}\right)=u_{\mathrm{b}}\left(1-\frac{a k}{\mathrm{e}}\right) .
$$

From $z=z_{\min }$ downwards to the bed the horizontal velocity increases. Note that, since $a k \ll 1$, it follows that $z_{\min } \gg a$.

Extrusion flow, a term introduced by Demorest (1941, 1942), has been a subject of some debate in the glaciological literature. On theoretical grounds, it can easily be shown that a global extrusion flow, that is an increase of the horizontal velocity with depth throughout an entire glacier, is impossible since the overlying mass will then experience a force in the main direction of flow, which is not counterbalanced by any other force, leading to an accelerating velocity (Nye, 1952). There are, on the other hand, claims of extrusion flow having been directly observed by borehole deformation measurements (Hooke and others, 1987) and by observations within subglacial caves close to the bedrock interface (Carol, 1947). Extrusive flow has also been observed within subglacial sediments (Blake and others, 1992). Arguments supporting (global) extrusion flow based on mass-balance measurements have also been given (Streiff-Becker, 1938; Seligman, 1947).

Morland (1976a) derived second-order solutions for flow over bedrock undulations using boundary conditions (1) and (2), and calculated explicit solutions valid along the bed line of a sinusoidal bed. Solutions based on his work, which are valid for the half-space above the bed, are given below and discussed.

Meyssonnier (1983) and Schweizer (1989) did FE calculations of flow over a sinusoidal bed. Meyssonnier obtained, in some of his numerical calculations, a point of maximum relative horizontal velocity that was situated above the peak of the sine wave, and some of Schweizer's calculations show a point of relative horizontal velocity minimum situated above the trough of the sine wave.

\section{SECOND-ORDER SOLUTIONS FOR A GRAVITY- DRIVEN FLOW}

Morland (1976a) incorporated gravity as the driving force of the motion and calculated terms to second order in $\varepsilon$. For the special case of a sinusoidal bed, he gave expressions valid for the pressure field and for the velocity components along the bed line, i.e. at $z=0$. Using Morland's results, one can calculate the velocity and the stress field as functions of $x$ and $z$, only somewhat laborious work is involved. All equations in this section follow from Morland (1976a).

The basal sliding velocity $u_{\mathrm{b}}$ is

$$
\begin{aligned}
u_{\mathrm{b}} & =\frac{\tau_{\mathrm{b}}}{\eta \varepsilon^{2} k} \frac{\left(\bar{w}^{2}+1\right)}{\bar{w}^{2}} \\
& =\frac{\tau_{\mathrm{b}}}{\eta \varepsilon^{2}}\left(\frac{1}{k}+\frac{1}{k_{*}} \frac{k}{k_{*}}\right),
\end{aligned}
$$

where the controlling wave number $k_{*}$ is defined by Equation (17), and $\bar{w}$ is defined as $\bar{w}:=k_{*} / k$. Table 1 compares the notation that is used here with the notation of several other authors.

If $k / k_{*} \ll 1$, the effects of regelation are negligible and the basal sliding velocity is given by

$$
u_{\mathrm{b}}=\frac{\tau_{\mathrm{b}}}{\eta k \varepsilon^{2}} .
$$

On the other hand, if $k / k_{*} \gg 1$, which is the pure regelation limit,

$$
u_{\mathrm{b}}=\frac{\tau_{\mathrm{b}} k}{\eta \varepsilon^{2} k_{*}^{2}} .
$$

$u_{\mathrm{b}}$ has a minimum at $k=k_{*}$. For a given amplitude-towavelength ratio the largest part of the drag is contributed by the Fourier components of the bed with wavelengths around $\lambda_{*}$, where $\lambda_{*}=2 \pi / k_{*}$ is given by Equation (15) (Nye, 1969). Note that

$$
u_{\mathrm{b}} \beta_{1}=\frac{\tau_{\mathrm{b}}}{\eta \varepsilon^{2} k},
$$

where

$$
\beta_{1}:=\frac{\bar{w}^{2}}{\bar{w}^{2}+1} .
$$

Equation (9) is a useful relation that can be used to eliminate the sliding velocity from the following equations.

The velocity field is given by

$$
\begin{aligned}
v_{x}(x, z)= & u_{\mathrm{b}}+\frac{\tau_{\mathrm{b}} h}{2 \eta}\left[1-\left(1-\frac{z}{h}\right)^{2}\right] \\
& +u_{\mathrm{b}} \beta_{1} k z \mathrm{e}^{-k z}\left(\sin k x-A_{\mathrm{m}} \cos k x\right) \varepsilon \\
+u_{\mathrm{b}} & \beta_{1} \mathrm{e}^{-2 k z}\left(\cos 2 k x+A_{\mathrm{m}} \sin 2 k x\right)\left(\frac{1}{4}-\frac{k z}{2}\right) \varepsilon^{2} \\
& +O\left(\varepsilon^{3}\right)
\end{aligned}
$$

and

$$
\begin{aligned}
v_{z}(x, z)= & u_{\mathrm{b}} \beta_{1} \mathrm{e}^{-k z}\left(\cos k x+A_{\mathrm{m}} \sin k x\right)(1+k z) \varepsilon \\
& +\frac{1}{2} u_{\mathrm{b}} \beta_{1} k z \mathrm{e}^{-2 k z}\left(\sin 2 k x-A_{\mathrm{m}} \cos 2 k x\right) \varepsilon^{2} \\
& +O\left(\varepsilon^{3}\right),
\end{aligned}
$$

where $A_{\mathrm{m}}$ is defined through Equation (18), and $h$ is the mean glacier thickness.

Table 1. Notation used here and that used by several different authors. $k_{*}$ is the controlling wave number and $\lambda_{*}$ the transition wavelength, $\lambda_{*}=2 \pi / k_{*}$, with $k_{*}=\sqrt{L / 2 \eta C_{0}\left(K_{\mathrm{I}}+K_{\mathrm{B}}\right)} \cdot r=a / \lambda$ is the single wavelength roughness and $\varepsilon:=$ $a k=2 \pi r$ is the (local bed) slope number. $L$ is the latent heat of fusion per unit volume of ice

$$
\text { This paper }
$$

Nye $(1969,1970)$

Kamb (1970),

Lliboutry (1987)

Morland (1976a)

\begin{tabular}{lllll}
\hline & & & $w_{*}$ & $1 / \bar{\lambda}_{*}$ \\
$k_{*}$ & $k_{*}$ & $l_{0}$ & $2 \pi \bar{\lambda}_{*}$ \\
$\lambda_{*}$ & $2 \pi / k_{*}$ & $\lambda_{0}$ & $2 \pi / w_{*}$ & $L$ \\
$k$ & $L$ & $H$ & $\rho L$ & $k$ \\
$r$ & $k$ & $h$ & $w$ & $\varepsilon / 2 \pi$ \\
\hline
\end{tabular}


For the special case of $z=0$, Equations (1la) and (11b) reduce to solutions for the velocity field along the bed line given earlier by Morland (1976a). Note that for $z=0$ the first-order term in Equation (1la) vanishes.

Equations (1la) and (11b) lead to the following expressions for the strain rates:

$$
\begin{aligned}
\dot{\epsilon}_{x x}= & u_{\mathrm{b}} \beta_{1} z k^{2} \mathrm{e}^{-k z}\left(\cos k x+A_{\mathrm{m}} \sin k x\right) \varepsilon \\
& +u_{\mathrm{b}} \beta_{1} k \mathrm{e}^{-2 k z}\left(\sin 2 k x-A_{\mathrm{m}} \cos 2 k x\right)\left(k z-\frac{1}{2}\right) \varepsilon^{2} \\
& +O\left(\varepsilon^{3}\right),
\end{aligned}
$$

and

$$
\begin{aligned}
\dot{\epsilon}_{x z}= & \frac{1}{2}\left(1-\frac{z}{h}\right) a^{2} k^{3} u_{\mathrm{b}} \\
& +u_{\mathrm{b}} \beta_{1} z k^{2} \mathrm{e}^{-k z}\left(A_{\mathrm{m}} \cos k x-\sin k x\right) \varepsilon \\
& +u_{\mathrm{b}} \beta_{1} k \mathrm{e}^{-2 k z}\left(k z-\frac{1}{2}\right)\left(\cos 2 k x+A_{\mathrm{m}} \sin 2 k x\right) \varepsilon^{2} \\
& +O\left(\varepsilon^{3}\right) .
\end{aligned}
$$

The second invariant of the strain-rate tensor is then found to be

$$
\begin{aligned}
\dot{\mathrm{II}}^{2}= & \frac{1}{4}\left(1-\frac{z}{h}\right)^{2} a^{4} k^{6} u_{\mathrm{b}}{ }^{2} \\
& +u_{\mathrm{b}}{ }^{2} \beta_{1}\left(1-\frac{z}{h}\right)\left(A_{\mathrm{m}} \cos k x-\sin k x\right) z k^{5} a^{2} \mathrm{e}^{-k z} \varepsilon \\
& +u_{\mathrm{b}}{ }^{2} \beta_{1}{ }^{2}\left(A_{\mathrm{m}}{ }^{2}+1\right) z^{2} k^{4} \mathrm{e}^{-2 k z} \varepsilon^{2} \\
& +u_{\mathrm{b}}{ }^{2} \beta_{1}\left(1-\frac{z}{h}\right)\left(k z-\frac{1}{2}\right) \\
& \cdot\left(A_{\mathrm{m}} \sin 2 k x+\cos 2 k x\right) a^{2} k^{4} \mathrm{e}^{-2 k z} \varepsilon^{2} \\
& +u_{\mathrm{b}}{ }^{2} \beta_{1}{ }^{2}\left(A_{\mathrm{m}}{ }^{2}+1\right)(2 k z-1) \sin k x z k^{3} \mathrm{e}^{-3 k z} \varepsilon^{3} \\
& +u_{\mathrm{b}}{ }^{2} \beta_{1}{ }^{2}\left(k z-\frac{1}{2}\right)^{2}\left(A_{\mathrm{m}}{ }^{2}+1\right) k^{2} \mathrm{e}^{-4 k z} \varepsilon^{4} \\
& +O\left(\varepsilon^{6}\right) .
\end{aligned}
$$

Equation (17) shows that, to second order, $\dot{\epsilon}_{\mathrm{II}}$ depends on both $x$ and $z$ and not only on $z$ as is the case in the firstorder Nye/Kamb solution.

Finally, the pressure distribution is given by

$$
\begin{aligned}
p(x, z)= & p_{\mathrm{a}}+\rho g \cos \alpha\left(1-\frac{z}{h}\right) \\
& +2 u_{\mathrm{b}} \beta_{1} \eta k \mathrm{e}^{-k z}\left(\cos k x+A_{\mathrm{m}} \sin k x\right) \varepsilon \\
& +u_{\mathrm{b}} \beta_{1} \eta k \mathrm{e}^{-2 k z}\left(\sin 2 k x-A_{\mathrm{m}} \cos 2 k x\right) \varepsilon^{2} \\
& +O\left(\varepsilon^{3}\right) .
\end{aligned}
$$

These expressions can be used to calculate the flow and the sliding velocity for a general bed geometry as long as $\varepsilon \ll 1$.

\section{The effect of regelation on the flow field}

Regelation is only important at wavelengths comparable to or smaller than the transition wavelength $\lambda_{*}$ (Weertman, 1957, 1964, 1979; Nye, 1969; Kamb, 1970), where

$$
\lambda_{*}=\sqrt{\frac{8 \pi^{2} \eta C_{0}\left(K_{\mathrm{I}}+K_{\mathrm{B}}\right)}{L}},
$$

and where $K_{\mathrm{I}}$ and $K_{\mathrm{B}}$ are the thermal conductivities of the ice and bed, respectively.

Two parameters $\left(\bar{w}\right.$ and $\left.A_{\mathrm{m}}\right)$ enter the flow solutions (Equations (1la) and (11b)) that describe the relative importance of regelation to viscous flow (Morland, 1976a). $\bar{w}$ is the ratio of the bed wavelength to the transition wavelength, i.e.

$$
\bar{w}^{2}:=\left(\frac{\lambda}{\lambda_{*}}\right)^{2}=\left(\frac{k_{*}}{k}\right)^{2}=\frac{L}{2 k^{2} \eta C_{0}\left(K_{\mathrm{I}}+K_{\mathrm{B}}\right)},
$$

where $k_{*}$ is the controlling wave number, given by

$$
k_{*}^{2}=\frac{L}{2 \eta C_{0}\left(K_{\mathrm{I}}+K_{\mathrm{B}}\right)} .
$$

In the no-regelation limit $\beta_{1}=1$ ( $\beta_{1}$ is defined by Equation (10) ) and in the pure-regelation limit $\beta_{1}=0 . A_{\mathrm{m}}$ is given by (Morland, 1976a)

$$
\begin{aligned}
A_{\mathrm{m}} & =\frac{\left(K_{\mathrm{I}}+K_{\mathrm{B}}\right) \cos (\alpha) \rho g C_{0}+2 Q}{L u_{\mathrm{b}}} \\
& =\frac{a^{2} k^{3} \eta}{L}\left(\frac{C_{0}\left(K_{\mathrm{I}}+K_{\mathrm{B}}\right) \cot (\alpha)}{h}+\frac{2 Q}{\tau_{\mathrm{b}}}\right) .
\end{aligned}
$$

The effect of freezing and melting on the flow field is negligible if $A_{\mathrm{m}} \ll 1$ and that is almost always the case (Morland, 1976a), which is the reason for ignoring the effect of regelation on the flow field in the following discussion of the properties of Equations (1la) and(1lb).

\section{Dimensionless form of the flow solutions}

For the following discussion, it is of advantage to rescale the dimensional quantities and to put the equations in a dimensionless form. To this end, dimensionless vertical and horizontal length scales, denoted by capital letters, are defined by

$$
X:=k x \quad \text { and } \quad Z:=k z,
$$

where the wave number $k$ is used as a scaling factor. The velocity field is scaled by the sliding velocity, so that

$$
V_{X}:=\frac{v_{x}}{u_{\mathrm{b}}} \quad \text { and } \quad V_{Y}:=\frac{v_{y}}{u_{\mathrm{b}}} .
$$

The dimensionless parameters which enter the problem are the slope parameter $\varepsilon$ and the thinness parameter $\delta:=$ $(k h)^{-1}$. Regelation will be ignored so that the following discussion is only valid for $\lambda \gg \lambda_{*}$.

Using the above-defined scalings, the velocity field is

$$
\begin{aligned}
& V_{X}(X, Z)=1+\frac{\varepsilon^{2}}{2 \delta}\left(1-(1-\delta Z)^{2}\right) \\
&+\varepsilon Z \mathrm{e}^{-Z} \sin X \\
&+\varepsilon^{2} \mathrm{e}^{-2 Z}\left(\frac{1}{4}-\frac{Z}{2}\right) \cos 2 X+O\left(\varepsilon^{3}\right)
\end{aligned}
$$

and

$$
\begin{aligned}
V_{Z}(X, Z) & =\varepsilon(1+Z) \mathrm{e}^{-Z} \cos X \\
& +\frac{1}{2} \varepsilon^{2} Z \mathrm{e}^{-2 Z} \sin 2 X+O\left(\varepsilon^{3}\right),
\end{aligned}
$$

where use has been made of Equation (7).

\section{OVERALL FEATURES OF THE HORIZONTAL VELOGITY FIELD}

Before going into a somewhat tedious mathematical discussion of the properties of the flow field, let us look at some contour plots of the horizontal velocity field to get an overall idea of the flow perturbations caused by the sinusoidal bed.

Figure 2 depicts $V_{X}(X, Z)$ as a function of $X$ and $Z$, for $\varepsilon=0.01$ and $\delta=0$, according to Equation (1la). The bed line is flat, since in the mathematical solution the sinusoidal bed profile has been projected on to the line $Z=0$. Note that $(X / \pi, Z)=\left(\frac{1}{2}, 0\right)$ and $(X / \pi, Z)=\left(\frac{3}{2}, 0\right)$ correspond, respectively, to the peak and the trough of the sinusoid. The most conspicuous features of the figure are the stationary points situated above the peak and the trough of the sinusoidal curve at $Z \approx 1$. 


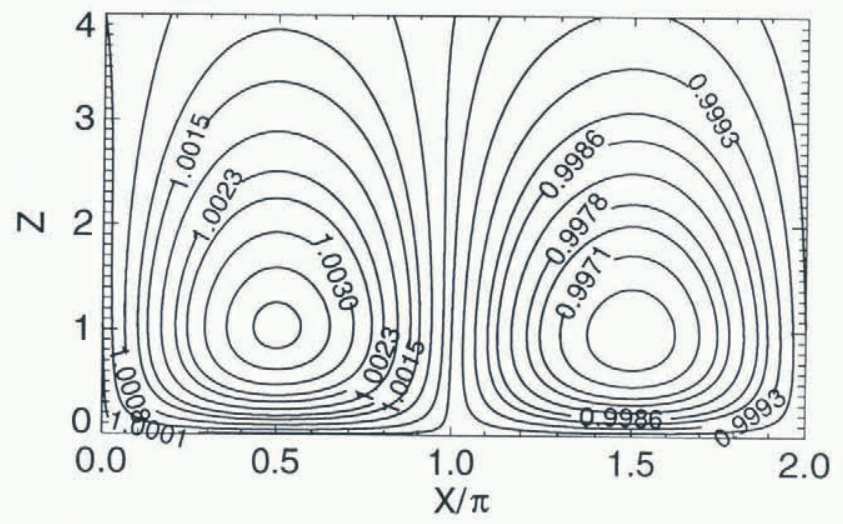

Fig. 2. The horizontal velocity, $V_{X}$, as a function of $X$ and $Z$ for $\varepsilon=0.01$ and $\delta=0$. The velocity maximum at $X / \pi=\frac{1}{2}$ and $Z \approx 1$ is referred to in the text as $U_{\pi / 2}^{\max }$. The minimum velocity at $X / \pi=\frac{3}{2}$ and $Z \approx 1$ is called $U_{3 \pi / 2}^{\min }$.

In Figure 3, the value of $\varepsilon$ has been changed from $\varepsilon=$ 0.01 to $\varepsilon=0.1$ as compared to Figure 2. The effect of this increase in $\varepsilon$ is to move the local maximum of the horizontal velocity field upward away from the bed, and the minimum of the velocity field down towards the bed. The amplitudes of the velocity perturbations are also considerably larger. Furthermore, a saddle point, where there is a local maximum in horizontal direction but a local minimum in vertical direction, can be seen above the velocity maximum at $X / \pi=\frac{1}{2}$.

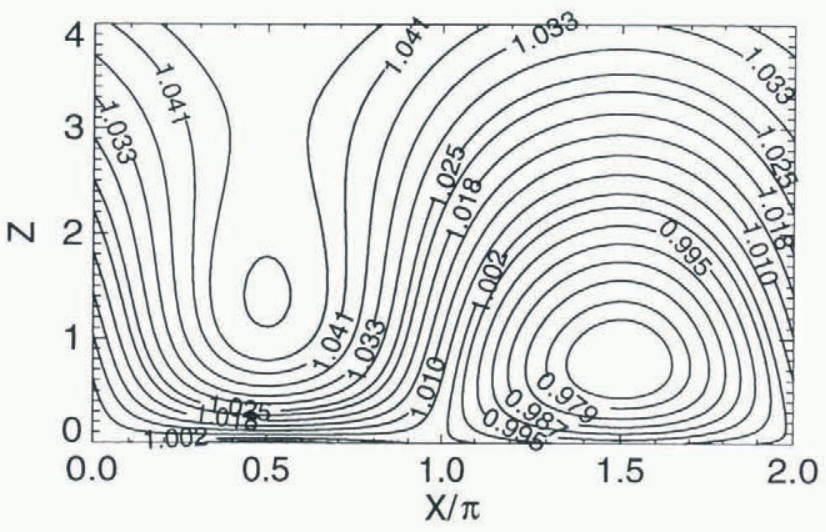

Fig. 3. $V_{X}$ as a function of $X$ and $Z$ for $\varepsilon=0.1$ and $\delta=0$. $U_{\pi / 2}^{\max }$ has moved upwards and $U_{3 \pi / 2}^{\min }$ downwards with respect to Figure 2. The point $U_{\pi / 2}^{\text {saddle }}$ can also be seen. As $\varepsilon$ increases further, $U_{\pi / 2}^{\max }$ and $U_{\pi / 2}^{\text {saddle }}$ move towards $Z=Z_{\text {crit }} \approx 1.98$, which they reach for $\varepsilon=\varepsilon_{\pi / 2}^{\text {crit }} \approx 0.138$. Simultaneously, $U_{3 \pi / 2}^{\min }$ moves downwards and reaches $Z=0$ for $\varepsilon=\varepsilon_{3 \pi / 2}^{\text {crit }}=\frac{1}{2}$

Increasing the value of $\varepsilon$ even further, as has been done in Figure 4, where $\varepsilon=\frac{1}{2}$, brings the minimum towards the bed line. The maximum point and the saddle point at $X / \pi=\frac{1}{2}$ have disappeared. As will be shown below, the maximum point and the saddle point cancel each other for $\delta=0$ at $\varepsilon \approx 0.138$.

The effect of changing the value of $\delta$ somewhat on the horizontal velocity field can seen by comparing Figure 5 , where $\varepsilon=0.1$ and $\delta=0.1$, to Figure 3, where $\varepsilon=0.1$ but $\delta=0.0$. The velocity maximum moves slightly towards the bed line and the saddle point further away from the bed as $\delta$ is increased from 0 to 0.1 . Increase in $\delta$ causes the local minimum of the horizontal velocity field to move away from the bed line.

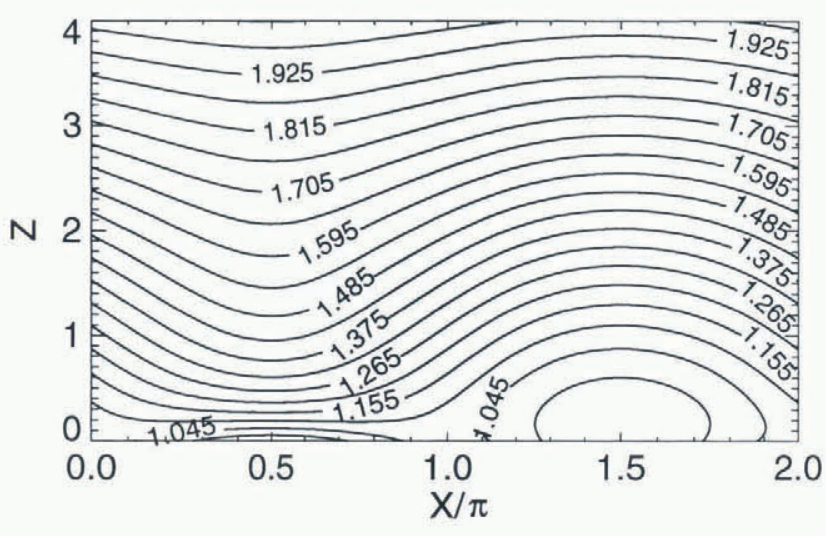

Fig. 4. $V_{X}$ as a function of $X$ and $Z$ for $\varepsilon=0.5$ and $\delta=0$. The points $U_{\pi / 2}^{\max }$ and $U_{\pi / 2}^{\text {saddle }}$ can no longer be seen and point $U_{3 \pi / 2}^{\min }$ is at $Z=0$.

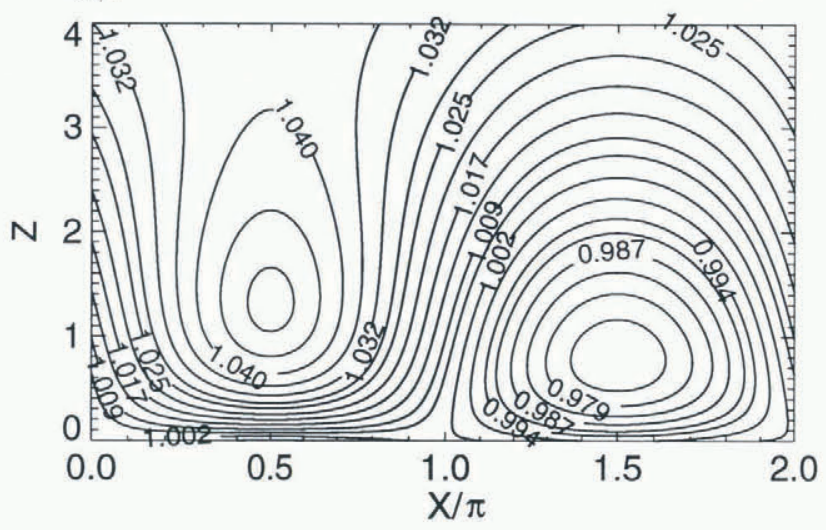

Fig. 5. $V_{X}$ as a function of $X$ and $Z$ for $\varepsilon=0.1$ and $\delta=0.1$. By comparing this figure with Figure 3 , the influence of $\delta$ can be seen.

One of the interesting features of Figures 2, 3 and 5 is that above the velocity maximum and below the velocity minimum a region where the horizontal velocity increases with depth (extrusion flow) is found. In the next section, the exact conditions under which extrusion flow develops are determined. Since the following discussion is somewhat tedious, the reader who is not interested in the fine details of the matter may find it better to skip the next section and read the summary of the results given in the last section of the paper.

\section{EXTRUSION FLOW}

It is of particular interest to know when extrusion flow occurs according to Equation (1la). This question can be answered by investigating when $V_{X}(X, Z)$ has a local maximum or minimum for $Z>0$. A necessary criterion for a stationary point of $V_{X}(X, Z)$ is that $\nabla V_{X}(X, Z)=0$.

\section{The horizontal positions of the stationary points}

Differentiation of Equation (1la) gives

$$
\begin{aligned}
\frac{\partial V_{X}}{\partial X}(X, Z) & =\varepsilon Z \mathrm{e}^{-Z} \cos X \\
& +\varepsilon^{2}\left(\frac{1}{2}-Z\right) \mathrm{e}^{-2 Z} \sin 2 X,
\end{aligned}
$$

and shows that $\partial V_{X}(X, Z) / \partial X=0$ has as solutions $X=$ $\pi / 2$ and $X=3 \pi / 2$ with no restrictions on $Z$. (It is to be understood that because of the periodicity of the sine func- 
tion, an integer multiple of $2 \pi$ can always be added to the values of $X$, although it will not be explicitly so written.

There is another interesting set of solutions given by

$$
\sin X=\frac{Z \mathrm{e}^{Z}}{\varepsilon(1-2 Z)} .
$$

It can be shown that this solution set is a second-order effect which is only important in the immediate vicinity of the bed line. This solution branch will not be discussed here further. The interested reader can find a detailed analysis of this solution set out in Gudmundsson (1994a). Ignoring this second-order effect there are, hence, only two stationary points. One is located above the peak of the sinusoidal curve, at $X=\pi / 2$, and can be shown to be a point of maximum velocity with respect to $X$. The other stationary point is situated above the trough, at $X=3 \pi / 2$, and is a point of minimum horizontal velocity with respect to $X$.

\section{The vertical positions of the stationary points}

Differentiating $V_{X}(X, Z)$ with respect to $Z$ and setting the resulting expression equal to zero gives

$$
1-\delta Z=\frac{1}{\varepsilon}(Z-1) \mathrm{e}^{-Z} \sin X+(1-Z) \mathrm{e}^{-2 Z} \cos 2 X .
$$

The interesting cases to be considered are $X=\pi / 2$ and $X=3 \pi / 2$, but there are also solutions at $X=0$ and $X=\pi$ with $Z=0$. These two solution points, situated at the bedrock interface, are saddle points, where the horizontal velocity obtains a maximum with respect to $X$ but a minimum with respect to $Z$. The existence of these points is a secondorder effect (Gudmundsson, 1994b) and they will not be discussed further.

\section{Stationary points situated above the peak of a sinusoid}

Let us begin with the case $X=\pi / 2$ in Equation (24) and see if there is a solution to the resulting equation

$$
\underbrace{1-\delta Z}_{=: L(\pi / 2, Z, \delta)}=\underbrace{\frac{1}{\varepsilon}(Z-1) \mathrm{e}^{-Z}+(Z-1) \mathrm{e}^{-2 Z}}_{=: R(\pi / 2, Z, \varepsilon)} .
$$

This is a non-linear equation that does not have a solution in a closed form. By plotting the lefthand side $(L(\pi / 2, Z, \delta))$ and the righthand side $(R(\pi / 2, Z, \varepsilon))$ separately, as is done in Figure 6, one sees that there will be a solution to Equation (25) if the righthand side, for at least one value of $Z$, becomes greater than, or equal to, $1-\delta Z$. This will happen if $\varepsilon$ is less than some particular value, that will now be called $\varepsilon_{\pi / 2}^{\text {crit }}$. There will, hence, only be stationary points at $X^{\pi / 2}=\pi / 2$ for some $\delta$ if $\varepsilon<\varepsilon_{\pi / 2}^{\text {crit }}$. To determine $\varepsilon_{\pi / 2}^{\text {crit }}$, write $\varepsilon$ in Equation (25) as a function of $Z$ and $\delta$ :

$$
\varepsilon(Z, \delta)=\frac{Z-1}{(1-\delta Z) \mathrm{e}^{Z}}+(1-Z) \mathrm{e}^{-Z} .
$$

Then $\varepsilon_{\pi / 2}^{\text {crit }}$ can be found by maximizing $\varepsilon(Z, \delta)$ given by Equation (26) subjected to $Z>0$ and $0 \leq \delta \ll 1$. By solving $\partial \varepsilon(Z, \delta) / \partial Z=0$ numerically, $\varepsilon_{\pi / 2}^{\text {crit }}$ can be calculated as a function of $\delta$. The details of these calculations can be found in Gudmundsson (1994b).

It is found that there are in general two stationary points along the line $X=\pi / 2$. An inspection of the determinant of the Hessian matrix of $V_{X}(X, Z)$ at the point $X=\pi / 2$ shows that one of the stationary points is a point of relative maximum. This velocity maximum will now be called $U_{\pi / 2}^{\max }$.

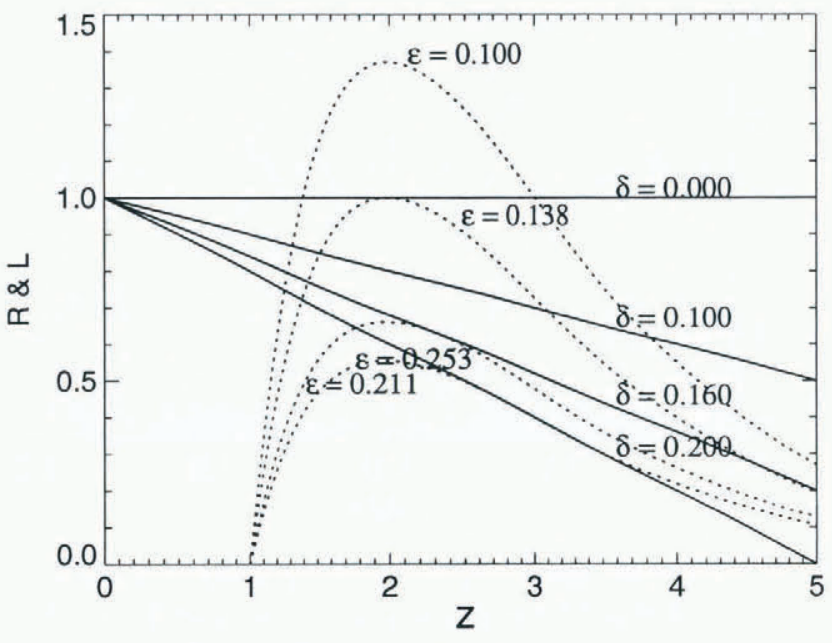

Fig. 6. $L(\pi / 2, Z, \delta)$ and $R(\pi / 2, Z, \varepsilon)$ as functions of $Z$. The solid lines represent the lefthand side of Equation (25) for a few different values of $\delta$ and the dotted lines show the righthand side of that same equation for different $\varepsilon$ values.

The other stationary point is a saddle point, where $V_{X}(X, Z)$ has a maximum with respect to $X$, but a minimum with respect to $Z$. The horizontal velocity component at this point will be called $U_{\pi / 2}^{\text {saddle }}$.

$\varepsilon_{\pi / 2}^{\text {crit }}$ is depicted in Figure 7 as a function of $\delta$. For $\delta=0$, $U_{\pi / 2}^{\max }$ and $U_{\pi / 2}^{\text {saddle }}$ exist as long as $\varepsilon<\varepsilon_{\pi / 2}^{\text {crit }} \approx 0.138$. The figure also shows that the value of $\varepsilon$, above which the horizontal velocity has no stationary points at $X=\pi / 2$, increases with $\delta$.

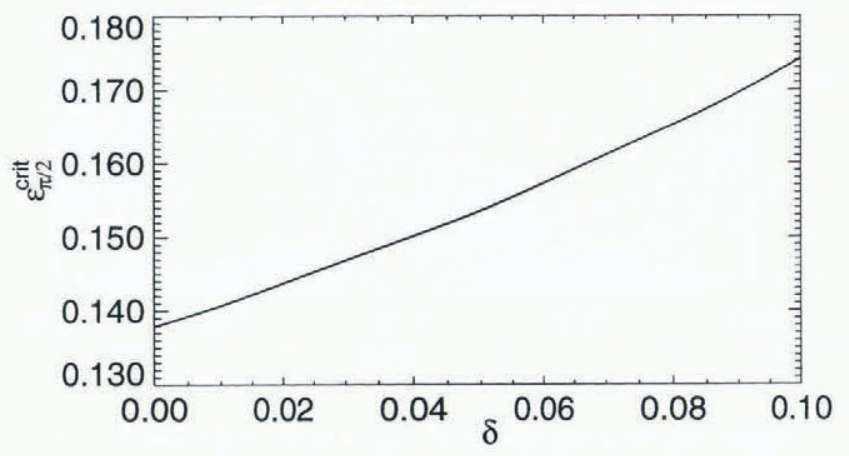

Fig. 7. $\varepsilon_{\pi / 2}^{\text {crit }}$ as a function of $\delta$. For $\varepsilon$ and $\delta$ values below the line there will be at least two solutions to Equation (25). One of these two solutions corresponds to a local maximum of the horizontal velocities above the peak of the sinusoidal bed and is called $U_{\pi / 2}^{\max }$. The other solution is situated above $U_{\pi / 2}^{\max }$ and corresponds to a saddle point of the horizontal velocities, where $V_{X}$ has a local maximum with respect to $X$ but a local minimum with respect to $Z$ and is called $U_{\pi / 2}^{\text {saddle }}$.

The changes in the vertical coordinates of both $U_{\pi / 2}^{\max }$ and $U_{\pi / 2}^{\text {saddle }}$ as $\varepsilon$ increases from zero are interesting. For $\varepsilon=0, U_{\pi / 2}^{\max }$ is at $Z=1$ and $U_{\pi / 2}^{\text {saddle }}$ at $Z=1 / \delta$. As $\varepsilon$ increases from zero towards $\varepsilon=\varepsilon_{\pi / 2}^{\text {crit }}, U_{\pi / 2}^{\max }$ moves upwards away from the bed, while $U_{\pi / 2}^{\text {saddle }}$ moves downward towards the bed line. Both stationary points then unite at $Z=$ $Z_{\text {crit }} \approx 1.98$ for $\varepsilon=\varepsilon_{\pi / 2}^{\text {crit }}$, and disappear (Gudmundsson, $1994 \mathrm{~b})$. These changes in the vertical coordinates of $U_{\pi / 2}^{\max }$ and $U_{\pi / 2}^{\text {saddle }}$ are illustrated in Figure 8 . 


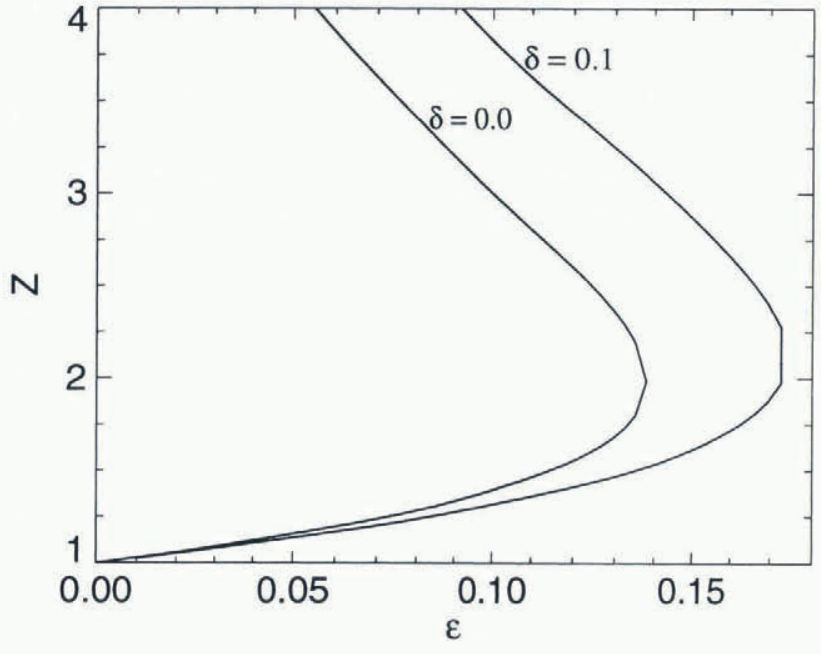

Fig. 8. The vertical positions of $U_{\pi / 2}^{\max }$ and $U_{\pi / 2}^{\text {saddle }}$ as functions of $\varepsilon$ for $\delta=0$ and $\delta=0.1 . Z_{\text {crit }}^{\pi / 2}$ and $\varepsilon_{\pi / 2}^{\text {crit }}$ are the points where the the slopes of the two lines are vertical. The branches above and below $Z_{\text {crit }}$ give the vertical positions of $U_{\pi / 2}^{\text {saddle }}$ and $U_{\pi / 2}^{\max }$, respectively. For $\varepsilon=\varepsilon_{\pi / 2}^{\text {crit }}$ there are no $U_{\pi / 2}^{\max }$ and $U_{\pi / 2}^{\text {saddle }}$ points.

The stationary points $U_{\pi / 2}^{\max }$ and $U_{\pi / 2}^{\text {saddle }}$ are not the only possible stationary points above the peak of the sinusoid which the horizontal velocity field can have. Figure 6 shows that the curvature of $R(\pi / 2, Z, \varepsilon)$ curves (dotted lines) can change, in which case the $L(\pi / 2, Z, \delta)$ curves (solid lines) can cross the dotted ones not only twice but three times. In this case, a third stationary point, $U_{\pi / 2}^{\mathrm{Up}}$, will be found along the line $X=\pi / 2$ together with $U_{\pi / 2}^{\pi / 2}$ and $U_{\pi / 2}^{\text {saddle }}$. It can be shown (Gudmundsson, 1994b) that the vertical position of $U_{\pi / 2}^{\mathrm{Up}}$ will always be above $Z=3$ and that it will in general be found close to the surface. This stationary point may appear because of the assumption, made in the derivation of the flow solutions, that the surface remains flat at all times. This assumption will only be approximately true when the bedrock is undulated. The properties of this stationary point will, hence, not be discussed further.

\section{Stationary points situated above the trough of a sinusoid}

The other possible $X$ value, beside $X=\pi / 2$, for a stationary point of $V_{X}(X, Z)$ is $X=3 \pi / 2$. Inserting $X=3 \pi / 2$ into Equation (24) leads to

$$
1-\delta Z=\mathrm{e}^{-Z}(1-Z)\left(\frac{1}{\varepsilon}-\mathrm{e}^{-Z}\right) .
$$

The lefthand side will always be greater than or equal to zero, because $Z$ must always be within the range $0 \leq Z \leq k h$. The $1 / \varepsilon-\mathrm{e}^{-Z}$ term on the righthand side will always be positive. There is therefore no solution possible for $Z>1$, but for $Z \leq 1$ there will always be a solution to Equation (27) as long as $\varepsilon \leq \frac{1}{2}$. This limiting value for $\varepsilon$ will be called $\varepsilon_{3 \pi / 2}^{\text {crit }}$, so that $\varepsilon_{3 \pi / 2}^{\text {crit }}=\frac{1}{2}$ for $\delta \ll 1$. For $\varepsilon \rightarrow 0$, the vertical coordinate of this stationary point will be at $Z=1$, and for $\varepsilon=\frac{1}{2}$ the point will be situated at $Z=0$ independently of the value of $\delta$. By looking at the determinant of the Hessian matrix, one finds that this stationary point is a point of relative minimum, and it will be referred to as $U_{3 \pi / 2}^{\min }$.

Equation (27) can be solved for $\varepsilon$, giving

$$
\varepsilon=\frac{1-Z}{(1-\delta Z) \mathrm{e}^{Z}+(1-Z) \mathrm{e}^{-Z}} .
$$

As $\delta$ varies, the position of $U_{3 \pi / 2}^{\min }$ changes somewhat. This is depicted in Figure 9, which shows the position of $U_{3 \pi / 2}^{\min }$ as a function of $\varepsilon$ for two different $\delta$ values. For a given $\varepsilon$ value and $\delta>0$, there will be two solutions for $Z$ of Equation (28). Only one of these solution points will be situated below $Z=1 / \delta$ (the other one is above the surface, and is a mathematical artifact) and it is this solution that is depicted in Figure 9.

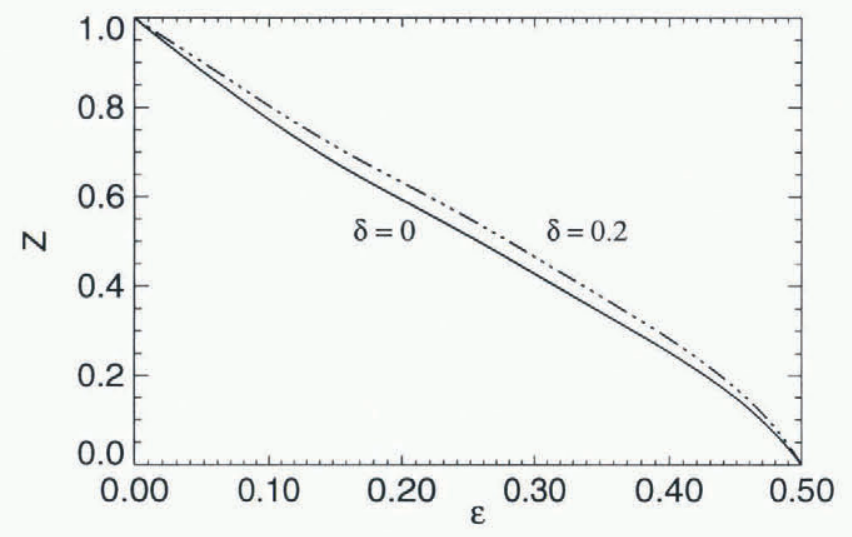

Fig. 9. The vertical position of $U_{3 \pi / 2}^{\min }$ as a function of $\varepsilon$ for $\delta=0$ and $\delta=0.2$. $U_{3 \pi / 2}^{\min }$ is the local minimum of the horizontal velocities above the trough of the sinusoidal curve (where $x=3 \pi / 2$ ). For $\varepsilon=0, U_{3 \pi / 2}^{\min }$ is situated at $Z=1$. As $\varepsilon$ increases, it approaches the bed and disappears at $\varepsilon=\frac{1}{2}$. Note that $\delta$ has almost no effect on the position of $U_{3 \pi / 2}^{\min }$.

Note that the perturbation approach is only valid as long as $\varepsilon \ll 1$. A value of $\varepsilon_{3 \pi / 2}^{\text {crit }}=\frac{1}{2}$ is, in this respect, rather large. It is therefore not clear whether the prediction that the stationary point above the trough of the sinusoid exists as long as $\varepsilon<\varepsilon_{3 \pi / 2}^{\text {crit }}=\frac{1}{2}$ can be trusted. Numerical approach seems to be the only possibility of getting a definitive answer.

The existence of the stationary points $U_{\pi / 2}^{\max }, U_{\pi / 2}^{\text {saddle }}$ and $U_{3 \pi / 2}^{\min }$ shows that there will be two regions of extrusion flow. One is at $X=\pi / 2$, which extends over the region that lies between the saddle point $\left(U_{\pi / 2}^{\text {saddle }}\right)$ and the maximum point $\left(U_{\pi / 2}^{\max }\right)$, and another one at $X^{\pi / 2}=3 \pi / 2$ that extends from the bed towards the point of local velocity minimum $\left(U_{3 \pi / 2}^{\min }\right)$. The region of extrusion flow along the line $X=\pi / 2$ will only exist if $\varepsilon<\varepsilon_{\pi / 2}^{\text {crit }}$ (see Fig. 7) and the extrusion flow at $X=3 \pi / 2$ only if $\varepsilon<\frac{1}{2}$.

\section{DISCUSSION}

\section{Comparison of the first- and the second-order velocity solutions}

The solution of Nye and of Kamb predicts, as said earlier, regions of extrusion flow. In this solution, the vertical positions of the local maximum of the horizontal velocity field above the peak of the sinusoid, $U_{\pi / 2}^{\max }$, and the local minimum above the trough, $U_{3 \pi / 2}^{\min }$, are always at $Z=1$ independent of the value of $\varepsilon$. The solutions of Nye and of Kamb have no saddle point. These findings of the first-order solution are reproduced by the second-order solution in the limit $\varepsilon \rightarrow 0$, as is to be expected. As $\varepsilon$ increases somewhat, there are, how- 
ever, profound differences between these two solutions. The inclusion of the effects of gravity on the flow field limits the occurrence of extrusion flow to a certain range of $\varepsilon$ values, and causes the vertical positions of the stationary points of the horizontal velocity field to depend on the amplitude-towavelength ratio of the bedrock undulations.

\section{Implications for borehole deformation}

The influence of the wavy nature of the bed on the flow is not largest at the bed but at some distance above it. Typically, this distance will be about 1 (or $z=1 / k$ ). Often the deformation of a borehole is used to get information on the rheological behaviour of the ice. One must interpret carefully the data from the lowest part of a borehole, since the exact form of the bedrock, which is usually not known, can have a large effect on the flow. Figure 3, for example, shows that the perturbed flow (the second and the third terms of Equation (21a)) dominates the gravity-driven plane flow (the first term of Equation (2la)) in the region $k z<3$.

The effect of extrusion flow on the deformation profile with depth will be to reverse it with respect to what one would expect from a simple plane-slab flow. Although the discussion here has been limited to one particular type of boundary condition (free-slip), extrusion flow can be expected to occur under other types of boundary conditions as well. It will, in general, be the lowest section of the borehole, within the vertical distance $\lambda / 2 \pi$, where $\lambda$ is the wavelength of a typical bedrock undulation, which will be affected by extrusion flow.

\section{A physical explanation for extrusion flow}

Upon reflection, it becomes evident that the vertical contraction and expansion of the ice close to the bed is responsible for the extrusion flow. At some distance sufficiently far above the bed, let us say at $z=z_{1}$, the ice moves parallel to the mean bed slope. For $k x=\pi / 2$, a high-pressure zone develops above the bed, which causes a Poiseuille flow, superimposed on the gravity-driven plane flow (GDPF) solution. The maximum of the Poiseuille velocity profile is at $\left(z_{1}-z_{0}\right) / 2$ and, if its decrease above that point is faster than the increase of the GDPF velocity profile, a velocity maximum will be found. Since the influence of the bed profile on the velocity field is (because of the factor $\mathrm{e}^{-k z}$ ) limited to a zone of height proportional to $1 / k, z_{1}$ will be proportional to $1 / k$ and one will expect the position of this maximum also to be proportional to $1 / k$. As a a matter of fact Equation (3a) has a maximum at $z=1 / k$ for $k x=$ $\pi / 2$. At $k x=3 \pi / 2$, the ice is expanded vertically and the Poiseuille flow profile reverses, causing a velocity minimum, again at $z=1 / k$.

The Nye and Kamb solution ignores GDPF and actually expresses nothing but this contraction and expansion due to the wavy nature of the bed. The velocity minimum and maximum therefore never disappears no matter how $\varepsilon$ is varied. If, on the other hand, the GDPF is present, it induces a subtle interplay between the Poiseuille flow and the GDPF. Therefore, only certain $\varepsilon$ values give rise to this interesting flow behaviour.

\section{Higher harmonics}

An interesting feature of Equations (1la) and (1lb) is the presence of the first harmonic of the fundamental period.
A simple physical argument shows that one must expect frequencies other than $\sin k x$ to appear in the solution, unless $\varepsilon \ll 1$, and that they will become more pronounced as $\varepsilon$ becomes larger.

Let us suppose that there were no higher harmonics in the expressions for $v_{x}$ and $v_{z}$, for all values of $\varepsilon$, so that

$v_{x}=u_{\mathrm{b}}+\hat{c}_{0} \sin k x$ and $v_{z}=\hat{c}_{1} \cos k x$, on $z=z_{0}$,

where $\hat{c}_{0}$ and $\hat{c}_{1}$ are some unknown constants. It follows that

$$
\frac{v_{x}}{v_{z}}=\frac{u_{\mathrm{b}}}{\hat{c}_{1} \cos k x}+\frac{\hat{c}_{0}}{\hat{c}_{1}} \tan k x .
$$

On the other hand, using the exact boundary condition (1) and $z_{0}=a \sin k x$ one obtains

$$
\frac{v_{x}}{v_{z}}=\frac{1}{a k \cos k x}
$$

at the base. Comparing Equation (30) to Equation (31) shows that $\hat{c}_{1}$ must be equal to $u_{\mathrm{b}} a k$ and that $\hat{c}_{0}$ has to be zero if expression (29) is to be true. But, this must be true for all $\varepsilon$ values, because Equation (3l) is always valid. On physical grounds, $\hat{c}_{0}=0$ can, however, be rejected; for high $\varepsilon$ values $v_{x}$ will certainly not be a constant on $z=z_{0}$, which means that the starting-point of Equation (29) must be incorrect. The velocity will therefore, in general, not be a single harmonic, although this may be approximately true for small roughness values.

\section{The stress field}

Using results from Morland (1976a), the two-dimensional stress field can be calculated. Detailed results have been given in Gudmundsson (1994b). It turns out that, in contrast to Equation (3f), the $\tau$ does depend on $x$ if second-order corrections are considered, as can in fact also been seen from Equation (13). Another interesting fact is that $\tau$ attains its largest value at $k z=1$ independently of $\varepsilon$.

\section{CONCLUSIONS}

Basal flow has been analysed using analytical solutions for a two-dimensional flow over bedrock undulations and criteria for extrusion flow have been given. Except for some secondorder effects, the horizontal velocity field can have at most three different stationary points close to a sinusoidal bed line:

If $0<\varepsilon<\frac{1}{2}$, there is a minimum point $\left(U_{3 \pi / 2}^{\min }\right)$ situated above the trough of the sinusoid (at $X=3 \pi / 2$ ). As $\varepsilon$ increases from zero towards $\varepsilon=\frac{1}{2}$, the vertical position of $U_{3 \pi / 2}^{\min }$ moves from $Z=1$ towards the bed line. As $\varepsilon=\frac{1}{2}$ is reached, $U_{3 \pi / 2}^{\min }$ hits the bed line and disappears. Since $V_{X}(X, Z)$ increases with depth below $U_{3 \pi / 2}^{\min }$ a zone of local extrusion flow is found close to the bed line as long as $U_{3 \pi / 2}^{\min }$ exists.

Above the peak of the sinusoid (at $X=\pi / 2$ ), there is a point of maximum surface-parallel velocity $\left(U_{\pi / 2}^{\max }\right)$, at which vertical position $Z$ is always within the bounds of $1<Z<Z_{\text {crit. }}$. It is found that $\lim _{\varepsilon \rightarrow 0} Z_{\text {crit }} \approx 1.98$ for $\delta=0 . U_{\pi / 2}^{\max }$ moves upwards away from the bed line with increasing $\varepsilon$.

Also, above the peak of the sinusoid (at $X=\pi / 2$ ), a saddle point $\left(U_{\pi / 2}^{\text {saddle }}\right)$ of $V_{X}(X, Z)$ develops, which exists for exactly the same range of $\varepsilon$ values as $U_{\pi / 2}^{\max }$. $U_{\pi / 2}^{\text {saddle }}$ is characterized by a maximum with respect to 
$X$ and a minimum with respect to $Z$ of $V_{X}(X, Z)$. Between the vertical positions of $U_{\pi / 2}^{\text {saddle }}$ and $U_{\pi / 2}^{\max }, V_{X}$ increases with depth.

It must be stressed that the extrusion flow discussed here, which is caused by bedrock undulations, is of local character. It should therefore possibly be called local extrusion flow, in order to distinguish it from extrusion flow encountered earlier in the glaciological literature, which was of global nature in the sense that it extended over a large area. The local extrusion flow does not add to the ice flux as global extrusion flow was thought to do. Local extrusion flow close to bedrock undulations is understandable in simple physical terms and must be expected to be a general feature of basal flow.

\section{ACKNOWLEDGEMENTS}

This research was supported by Swiss Science Foundation grant 20-29619.90. I thank A. Iken, C. F. Raymond, K. Hutter and D. Bahr for thorough reviews of the manuscript, and B. Hallet for bringing $\mathrm{H}$. Carol's paper on the formation of roches moutonnées to my attention.

\section{REFERENCES}

Blake, E., G. K. C. Clarke and M. C. Gérin. 1992. Tools for examining subglacial bed deformation. F. Glaciol., 38 (130), 388-396.

Carol, H. 1947. The formation of roches moutonnées. J. Glaciol., 1 2), 57-59.

Demorest, M. 1941. Glacier flow and its bearing on the classification of glaciers. Geol. Soc. Am. Bull., 52 (12), Part 2, 20242025.

Demorest, M. 1942. Glacier regimes and ice movements within glaciers. $\mathrm{Am}$. J. Sci., $240(1), 31-66$.

Fowler, A. C. 1979. A mathematical approach to the theory of glacier sliding. 7. Glaciol., 23 89), 131-141.

Fowler, A. C. 1981. A theoretical treatment of the sliding of glaciers in the absence of cavitation. Philos. Trans. R. Soc. London, Ser. A, 298 (1445), 637 685.

Gudmundsson, G. H. 1994a. Convergent glacier flow and perfect sliding over a sinusoidal bed. (Ph.D. thesis, Eidgenössische Technische Hochschule, Zürich, No. 10711.

Gudmundsson, G. H. 1994b. Glacier sliding over sinusoidal bed and the characteristics of creeping flow over bedrock undulations. Eidg. Tech. Hochschule, Zürich.Versuchsanst. Wasserbau, Hydrol. Glaziol. Mitt. 130.

Gudmundsson, G. H. 1997. Basal-flow characteristics of a non-linear flow sliding frictionless over strongly undulating bedrock. J. Glaciol., $43(143), 80-89$.

Hooke, R. LeB., P. Holmlund and N. R. Iverson. 1987. Extrusion flow demonstrated by bore-hole deformation measurements over a riegel, Storglaciären, Sweden. J. Glaciol., 33 (113), $72-78$.

Kamb, B. 1970. Sliding motion of glaciers: theory and observation. Rev. Geophys. Space Phys., 8 4), 673-728.

Lliboutry, L. A. 1987. Very slow flowes of solids: basics of modeling in geodynamics and glaciology. Dordrecht, etc., Martinus Nijhoff Publishers.

Meyssonnier, J. 1983. Ecoulement de la glace sur un lit de forme simple: expérience, modélisation, paramétrisation du frottement. (Ph.D. thesis, Université de Grenoble I.

Morland, L. W. 1976a. Glacier sliding down an inclined wavy bed. J. Glaciol., $17(77), 447-462$.

Morland, L. W. 1976b. Glacier sliding down an inclined wavy bed with friction. F. Glaciol., 17 (77), $463-477$.

Nye, J. F. 1952. Reply to Mr. Joel E. Fisher's comments, J. Glaciol., 2(11), 52 53.

Nye, J. F. 1969. A calculation on the sliding of ice over a wavy surface using a Newtonian viscous approximation. Proc. R. Soc. London, Ser. A, 311 (1506), $445-467$.

Nye, J. F. 1970. Glacier sliding without cavitation in a linear viscous approximation. Proc. R. Soc. London, Ser. A, 315(1522), 381-403.

Raymond, C. F. Unpublished. Numerical calculation of glacier flow by finite elements methods. Seattle, WA, University of Washington. Geophysics Program. Unpublished report for National Science Foundation Grant No. DPP74-19075.

Schweizer, J. 1989. Friction at the base of a glacier. Eidg. Tech. Hochschule, Zürich. Versuchsanst. Wasserbau, Hydrol. Glaziol. Mitt. 101.

Schweizer, J. and A. Iken. 1992. The role of bed separation and friction in sliding over an undeformable bed. J. Glaciol., 38(128), $77-92$.

Seligman, G. 1947. Extrusion flow in glaciers: a description of tests to prove the hypothesis and some details regarding its influence on glacial erosion. f. Glaciol., 1 (1), 12 21.

Streiff-Becker, R. 1938. Zur Dynamik des Firneises. Z Gletscherkd., 25 (1-2), $1-21$.

Weertman, J. 1957. On the sliding of glaciers. F. Glaciol., 3(21), 33-38.

Weertman, J. 1964. The theory of glacier sliding. F. Glaciol., 5 (39), 287-303,

Weertman, J. 1979. The unsolved general glacier sliding problem. J. Glaciol., $23(89,97-115$. 